\title{
Bronchoscopic Management of Tracheobronchial Stenosis Secondary to Granulomatosis with Polyangitis: A Case Report
}

Hari Kishan*, Supparerk Disayabutr and Jamsak Tscheikuna

Division of Respiratory Disease and Tuberculosis, Faculty of Medicine Siriraj Hospital, Mahidol University, Bangkok, Thailand

\begin{abstract}
Airway manifestations of Granulomatosis with Polyangitis (GPA) can be varied ranging from simple mucosal inflammation to critical tracheobronchial stenosis. Management of critical airway stenosis secondary to GPA is challenging. Significant airway compromise might require immediate evaluation and endoscopic or surgical intervention. Endoscopic interventions either in the form of balloon dilatation, electrosurgery, laser therapy, intralesional corticosteroid injection, topical application of mitomycin C, endoluminal spray cryotherapy or stent placement, remain the mainstay of treatment especially in patients who are deemed high risk and not a candidate for reconstructive surgery. We describe a case of tracheobronchial stenosis secondary to GPA, presenting with respiratory insufficiency requiring mechanical ventilator assistance on tracheostomy, successfully managed by endoscopic intervention combining bronchoscopic balloon dilatation and airway stent placement.
\end{abstract}

Keywords: Polyangitis; Tracheostomy; Critical airway

\section{Introduction}

Granulomatosis with Polyangitis (GPA), previously known as Wegener's Granulomatosis, is a form of anti-neutrophil cytoplasmic antibodies (ANCA)-associated vascuilitis predominantly involving the upper and lower respiratory tract and kidneys. Tracheobronchial involvement in GPA can have varied manifestations which include airway inflammation, mucosal granulomas, tracheobronchial stenosis, tracheal and endobronchial mass lesions (inflammatory pseudo tumors), tracheobronchomalacia and tracheoesophageal fistulae [1-3]. Tracheobronchial involvement is seen in nearly 30 to $50 \%$ of the cases $[1,4]$. The subglottic portion of the trachea is most commonly affected [5]. Management of tracheobronchial GPA is complex, including endoscopic and surgical approach. The management of lower tracheal and bronchial stenosis is less reported than subglottic stenosis in tracheobronchial GPA.

In this study, we described a case of GPA with tracheobronchial involvement causing respiratory failure who was successfully managed by bronchoscopic balloon dilatation and airway stent placement.

\section{Case Report}

A 57-year-old gentleman was previously diagnosed to have granulomatosis with polyangitis (GPA) since 2015. At diagnosis, he presented with right orbital cellulitis with peripheral ulcerative keratitis, left maxillary sinusitis and diffuse alveolar hemorrhage. He underwent right enucleation and the histopathology of the right eye globe showed focal fibrinoid necrosis of small blood vessels with multinucleated giant cells suspicious of vasculitis. He also underwent functional endoscopic sinus surgery (FESS) and the histopathology of the right nasal septal biopsy was consistent with necrotizing granulomatous inflammation. No causative pathogens were identified by cultures and histopathology. He had tested positive for PR3 antibodies and C-ANCA. He received tracheostomy due to prolonged intubation. He was successfully treated with plasmapharesis, pulse methylprednisolone and 10 cycles of intravenous cyclophosphamide pulse therapy. He was on maintenance treatment with azathioprine $50 \mathrm{mg} /$ day and prednisolone $20 \mathrm{mg} /$ day. After discharge, he had productive cough and occasional noisy breathing. He had dyspnea on exertion and could climb a flight of stairs. For this admission, he presented to the department of otorhinolaryngology with worsening dyspnea for 1 week with difficulty in expectoration. He had no fever. Physical examination revealed severe respiratory distress and paradoxical breathing with stridor and generalized rhonchi on both lungs. His chest X-ray showed no infiltrate. Complete blood count showed mild elevation of white blood cell count with neutrophil predominance. Blood chemistry and urinalysis were normal. After initiation of mechanical ventilation, his symptoms were improved but he still had inspiratory rhonchi on both lungs. Flexible bronchoscopy was performed to evaluate the central airway. Bronchoscopic findings included subglottic stenosis with small granulation tissue above the stoma of tracheostomy and diffuse mucosal inflammation of tracheobronchial trees. Mid to lower trachea were $70 \%$ stenosis due to mucosal swelling and tracheomalacia as shown in Figure 1. Left main bronchus and segmental bronchi showed $60 \%$ collapse during expiration due to bronchomalacia (Figure 1). He was referred to our department for stent placement as surgical reconstruction was not a favorable approach with respect to the extent of the lesions and patient's condition.

We subjected the patient to rigid bronchoscopy under general anesthesia. Tracheal dilatation was performed by using balloon dilatation and rigid bronchoscope. Left main bronchus was also dilated using balloon dilatation. A silicone stent was then placed at the lower trachea. The proximal end of the stent was just below the tip of tracheostomy tube and the distal end of the stent was above the carina as shown in Figure 2.

We were able to wean him off the ventilator support immediately after the intervention and maintained on oxygen collar via tracheostomy. There were no complications during intra-operative and immediate post-operative periods. His clinical condition improved significantly after intervention, and was maintained on azathioprine 50 $\mathrm{mg}$ /day and prednisolone $10 \mathrm{mg} /$ day. We were able to discharge him three days after the intervention. Surveillance flexible bronchoscopy

*Corresponding author: Hari Kishan, Division of Respiratory Disease and Tuberculosis, Faculty of Medicine Siriraj Hospital, Mahidol University, Bangkok, Thailand, Tel: 0241977578; E-mail: harikishang@icloud.com ; E-mail: harikishang@icloud.com

Received November 22, 2017; Accepted November 24, 2017; Published November 30, 2017

Citation: Kishan H, Disayabutr S, Tscheikuna J (2017) Bronchoscopic Management of Tracheobronchial Stenosis Secondary to Granulomatosis with Polyangitis: A Case Report. J Pulm Respir Med 7: 433. doi: 10.4172/2161-105X.1000433

Copyright: (C) 2017 Kishan H, et al. This is an open-access article distributed under the terms of the Creative Commons Attribution License, which permits unrestricted use, distribution, and reproduction in any medium, provided the original author and source are credited. 

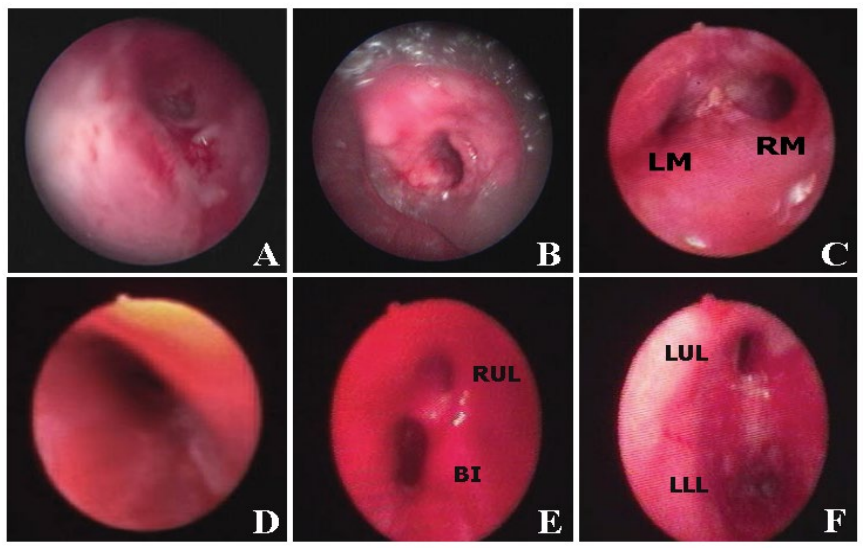

Figure 1: Bronchoscopic finding. (A) subglottic stenosis (B) tracheal stenosis with tracheomalacia at lower trachea distal to the tip of tracheostomy tube (C) mucosal inflammation with bronchomalacia at carina and opening of left main bronchus $(D)$ bronchomalacia at left main bronchus ( $E$ and $F$ ) mucosal inflammation at right and left main bronchus.

LM: Left Main Bronchus; RM: Right Main Bronchus; RUL: Right Upper Lobe Bronchus; BI: Bronchus Intermedius; LUL: Left Upper Lobe Bronchus; LLL: Left Lower Lobe Bronchus
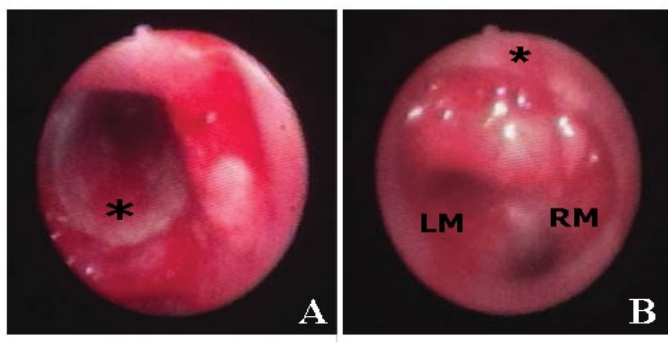

Figure 2: Silicone stent $\left({ }^{*}\right)$ was placed in lower trachea. (A) The proximal end of stent was just below the tip of tracheostomy $(B)$ The distal end of the stent was above the carina.

LM: Left Main Bronchus; RM: Right Main Bronchus

was performed at 4 weeks interval showed luminal patency with minimal secretions which were cleared.

\section{Discussion}

Granulomatosis with Polyangitis is a necrotizing granulomatous inflammation mainly involving the upper and lower respiratory tract. Immunosuppressive therapy remains the mainstay of treatment in patients with GPA. Tracheobronchial involvement often runs a course independent of other manifestations of GPA [6], and effective local treatment of airway lesions can lead to early tapering of systemic immunosuppression in selected patients. A spectrum of patients continues to develop symptoms of airway obstruction despite clinical remission induced by standard immunosuppressive regimens as evident in our case where remission induction was successful. Prompt recognition and airway management can lead to reduction in pulmonary morbidity and to prevent life threatening ventilatory and infective complications. Tracheobronchial stenosis may not always respond to systemic immunosuppressive therapy. The symptoms and overall general condition of patient and the severity of the stenosis should determine the appropriate treatment strategy on an individualized basis.

Therapeutic alternatives to standard medical therapy include resection and reconstructive surgery, tracheostomy, and endoscopic approach. Various endoscopic methods have been described in the literature, but there is no consensus statement on the optimal therapeutic approach. The different endoscopic techniques have been described including balloon dilatation, intralesional steroid injection [6], laser resection, endoluminal spray cryotherapy [7] and stent placement.

There is a divergence of opinion regarding the optimal endoscopic approach in patients with tracheobronchial GPA. Though surgical reconstruction remains the standard of care, it is not always feasible for the surgeon to resect and reconstruct, especially when the disease is not localized, as in our case where there is multilevel obstruction. Endoscopic dilatation is a minimally invasive procedure causing least insult to the airway epithelium and can be accomplished by serial rigid tube dilatation or balloon catheter. Some authors have shown that dilatation combined with intralesional corticosteroid injection, especially in cases of active tracheobronchial GPA would obviate the need for airway bypass surgery or stenting while preventing the long term complication of chronic airway scarring. Nouraei and colleagues has reported that the requirement for tracheostomy could be reduced from $40-50 \%$ to zero [6]. But this is not always possible, especially when the patients present acutely with life threatening obstruction. The use of airway stents to maintain airway patency in tracheobronchial GPA is also largely debated. Silicone stents are preferred because metal stents can be removed with great difficulty and may also penetrate to the adjacent tissue. In our case we used a silicone stent to maintain patency of the lower trachea after serial tracheal dilatation using balloon and rigid bronchoscope. Though there is a lot of controversies regarding the use of airway stents in tracheobronchial GPA, our case report demonstrates that silicone stent placement provide symptomatic relief and should be considered when other conservative methods fail to restore airway patency. Duam and colleagues observed that silicone stents provided persistent airway patency when compared to rigid tube dilatation and YAG laser therapy [8]. In our case, bronchoscopic balloon dilatation and stent placement led to significant clinical improvement, and we were able to wean the patient off the ventilator immediately following the procedure. Surveillance bronchoscopy one month later showed good luminal patency with very minimal secretions.

\section{Conclusion}

We suggest that bronchoscopic balloon dilatation and stent placement should be considered in patients with tracheobronchial GPA, presenting with acute respiratory failure when other conservative methods fail to maintain airway patency.

\section{References}

1. Lee KS, Kim TS, Fujimoto K (2003) Thoracic manifestation of Wegener's granulomatosis: CT findings in 30 patients. Eur Radiol 13: 43-51.

2. Polychronopoulos VS, Prakash UB, Golbin JM, Edell ES, Specks U (2007) Airway involvement in Wegener's granulomatosis. Rheum Dis Clin North Am 33: 755

3. Travis WD, Hoffman GS, Leavitt RY, Pass HI, Fauci AS (1991) Surgical pathology of the lung in Wegener's granulomatosis. Review of 87 open lung biopsies from 67 patients. Am J Surg Pathol 15: 315

4. Cordier JF, Valeyre D, Guillevin L (1990) Pulmonary wegener's granulomatosis: A clinical and imaging study of 77 cases. Chest 97: 906-912.

5. Screaton NJ, Sivasothy P, Flower CD, Lockwood CM (1998) Tracheal involvement in Wegener's granulomatosis: Evaluation using spiral CT. Clin Radiol 53: 809.

6. Nouraei SA, Obholzer R, Ind PW, Salama AD, Pusey CD, et al. (2008) Results of endoscopic surgery and intralesional steroid therapy for airway compromise due to tracheobronchial Wegener's granulomatosis. Thorax 63: 49-52. 
Citation: Kishan H, Disayabutr S, Tscheikuna J (2017) Bronchoscopic Management of Tracheobronchial Stenosis Secondary to Granulomatosis with Polyangitis: A Case Report. J Pulm Respir Med 7: 433. doi: 10.4172/2161-105X.1000433

Page 3 of 3

7. Bhora FY, Ayub A (2016) Treatment of Benign Tracheal Stenosis Using Endoluminal Spray Cryotherapy. JAMA Otolaryngol Head Neck Surg 142: 1082-1087.
8. Daum TE, Specks U, Colby TV, Edell ES, Brutinel MW, et al. (1995) Tracheobronchial involvement in Wegener's granulomatosis. Am J Respir Crit Care Med 151: 522-526. 